\title{
Virtual Reality Games: a study about the level of interaction $v s$. narrative and the gender in presence and cybersickness.
}

\author{
Guilherme Gonçalves \\ UTAD \\ Vila Real, Portugal \\ guilhermeg@utad.pt
}

\author{
Miguel Melo \\ INESC TEC \\ Porto, Portugal \\ mcmelo@inesctec.pt
}

\author{
Maximino Bessa \\ UTAD and INESC TEC \\ Vila Real and Porto, Portugal \\ maxbessa@utad.pt
}

\begin{abstract}
Virtual reality (VR) games have the potential to produce immersive experiences. To better explore the potential of VR games, it becomes necessary to understand what affects the player's presence in VR games. This work measures and compares the levels of presence and cybersickness in VR environments. Two games with different levels of interaction and narrative were compared. Presence and cybersickness were measured in a sample of 32 subjects using the IPQp questionnaire and a Portuguese version of the SSQ respectively. The results indicate that there were no differences in presence and cybersickness between the interaction and the narrative dimensions. To extend the study, the gender of participants was also considered an independent variable where we found significant differences in the metrics of presence and experienced realism, nausea and disorientation with female participants getting higher scores.
\end{abstract}

Keywords - virtual reality, games, presence, cybersickness, interaction, narrative, gender.

\section{INTRODUÇÃO}

Os sistemas de realidade virtual (RV) têm vindo a mostrar provas do seu enorme potencial ao longo dos anos. O seu crescimento deve-se também ao aumento do poder de processamento dos computadores permitindo a síntese de imagens e a interações entre o utilizador e o mundo virtual em tempo real[1]. A RV torna possíveis, de maneira mais imersiva, as atividades cognitivas e sensoriomotoras de um utilizador num mundo gerado por computador [2].

Os termos "Presença" e "Imersão" têm sido usados amplamente no campo da Realidade Virtual como uns dos pontos mais importantes da tecnologia [3]-[5]. A sensação de presença é considerado como um estado da consciência, a sensação psicológica de estar dentro de um mundo virtual [5][10]. Participantes que se sentem muito presentes deverão sentir o ambiente virtual como sendo uma realidade mais envolvente que o mundo físico ao seu redor e consequentemente assumir os ambientes virtuais como sítios visitados em vez de imagens visualizadas[6]. O conceito de presença é uma forma consensual de avaliar subjetivamente a eficácia dos ambientes virtuais.

978-1-5386-8228-9/17/\$31.00 C2018 IEEE
Para o efeito foram desenvolvidos vários questionários de presença tais como o PQ [11], IPQ [12] ou ITC-SOPI [13]. No desenvolvimento de aplicações de RV torna-se, portanto importante saber que fatores podem influenciar esta sensação de presença de maneira a otimizar os jogos e tirar o máximo proveito em termos de imersão.

Por outro lado, interação e narrativa são componentes fortes presentes nos jogos, pelo qual serão estudados de maneira a perceber como podem afetar a sensação de presença. Existem igualmente diferentes tipos de jogos focados em diferentes públicos alvo. Saber de que maneira o género do público alvo pode influenciar a presença é igualmente importante para o desenvolvimento de aplicações RV. A nível de presença, iremos neste trabalho investigar qual o papel da interação, narrativa e género.

Estudos indicam que a Interação tem um papel preponderante na experiência do utilizador com uma correlação positiva com a sensação de presença [14]-[17]. Existem várias noções de interação em ambientes virtuais: Slater e Usoh consideram a interação como a capacidade do utilizador de se deslocar e alterar o mundo virtual, ou seja, navegação e manipulação [18]. Zeltzer define interação como a habilidade de alterar parâmetros do ambiente virtual com resposta imediata [19]. Steuer caracteriza interação como a extensão até à qual os utilizadores podem modificar a forma e conteúdo de um ambiente em tempo real [8]. Em outros estudos [11], [20] resultados indicam que quanto maior a atenção e esforço exigido do jogador, mais presente este se sentirá.

Em relação à narrativa na sensação de presença, foi mencionado que um enredo que retire o utilizador da vida quotidiana para um mundo alternativo e independente proporcionará uma maior possibilidade aos utilizadores de se sentirem mais presentes neste [6]. Busselle e Bilandzic [21] afirmam que uma narrativa tem a capacidade de dirigir o foco de maneira a que o utilizador perca a noção do espaço envolvente ao transportá-lo para um mundo alternativo, ou seja, aumentando a sensação de presença.

Relativamente à dissimilitude entre géneros, estudos sugerem a existência de diferenças nos níveis de presença sentidos em ambientes imersivos [22] e pouco imersivos[23], [24]. Um estudo realizado por Melo et al. [25] revelou que, para ambientes virtuais sintetizados, as participantes 
experienciam maior realismo experienciado (uma subescala do questionário IPQ). Noutro estudo, envolvendo vídeos $360^{\circ}$ 2D/3D [26], resultados sugerem a inexistência de diferenças entre géneros nas métricas de presença.

Pela literatura podemos verificar que tanto as componentes de interação e narrativa têm o potencial de aumentar a sensação e presença. No entanto falta verificar se ambas componentes têm o mesmo peso ou qual delas representa um maior impacto na presença. Podemos também verificar pelos estudos realizados que a sensação de presença pode diferir nos seus níveis entre géneros.

Outro conceito pertinente no que diz respeito à utilização de sistemas de RV é o de cybersickness. Face à exposição à RV, por vezes os utilizadores manifestam sintomas parecidos com aqueles do motion sickness. Este fator pode prejudicar a experiência, ou até mesmo desencorajar as pessoas de usar tecnologias de RV. A sua avaliação pode ser realizada através de questionários como o SSQ [27] ou Pensacola Diagnostic Index [28]. Entre os sintomas possíveis temos a tensão ocular, dor de cabeça, palidez, suor, boca seca, desorientação, vertigens, náuseas, ataxia e vómitos [9], [29]. Estes sintomas podem surgir por vários motivos, no entanto, no caso da RV, geralmente o fator principal é o conflito criado entre o sistema vestibular e visual. Quando o equipamento de RV não consegue acompanhar os movimentos do utilizador, ou o ambiente virtual envia estímulos visuais que ultrapassem a capacidade do sistema de RV em dar o devido e coerente feedback visual, sintomas de cybersickness poderão surgir. Quanto maior o controlo do utilizador sobre as ações (ex. girar a cabeça, caminhar) menor será a probabilidade de cybersickness [30]. Os sintomas de cybersickness são temporários, no entanto pode existir a possibilidade de after effects onde podem surgir sintomas nas seguintes horas, o que pode representar perigo para os utilizadores de RV [9]. É importante entender como as componentes dos jogos a serem estudadas (interação e narrativa) e diferenças entre géneros podem influenciar este aspeto. Tornou-se, portanto, crítico tentar evitar ou minimizar ao máximo estes efeitos secundários de maneira a evitar que estes influenciem o comportamento e bem-estar dos participantes durante e após a experiência virtual.

Estudos indicam que as diferenças de género influenciam a suscetibilidade ao cybersickness [9], [31]. Reason e Brand [32] reportam que o género feminino é mais suscetível que o masculino em todas as formas de motion sickness. Noutro estudo, os resultados indicam que a sensação de presença está correlacionada positivamente com cybersickness [33]. Apesar do estado de arte indicar que géneros diferem entre si no cybersickness, existe igualmente estudos que concluem a inexistência destas diferenças [26]. O facto da tecnologia de RV ter evoluído e estar mais madura poderá alterar este panorama pelo que é importante incluir esta componente no estudo.

Com isto estabelecemos dois objetivos específicos neste trabalho: estudar a influência da interação e narrativa de dois jogos de RV na sensação de presença e de cybersickness; estudar a influência do género na sensação de presença e cybersickness. Será que um jogo focado apenas na interação com o mundo virtual terá maior influência na presença do participante do que um jogo focado numa narrativa cativante? Será que ambos os fatores são igualmente importantes? Deveremos apostar mais na interação para com o ambiente virtual e o ambiente virtual para connosco, ou numa narrativa mais imersiva? Será que ao ter uma componente mais pesada de interação iremos ter em contrapartida mais cybersickness devido à maior quantidade de movimentos requeridos? Existirá diferenças entre géneros na presença e cybersickness reportados? Para estudar estas questões, este trabalho irá considerar dois jogos representativos dos dois cenários identificados: um jogo com muita interação e pouca narrativa (doravante referido como $\mathrm{I}+\mathrm{N}-$ ) e outro com pouca interação e boa narrativa (doravante referido como I-N+) (Tabela I).

A equipa de investigação especula que a interação terá um peso maior na sensação de presença que uma boa narrativa pelo fato de requerer do utilizador mais ações e maior atenção ao que se está a passar no ambiente virtual. Esta especulação é suportada por estudos como o de Witmer e Singer [11] que refere que quanto maior for o controlo que o jogador tem no ambiente virtual maior será a presença. Conforme Zahorik and Jenison [34]: "presence is tied to action in the environment". Presumimos que tal irá envolver mais o utilizador do que um ambiente focado na narrativa. Esperamos então que a condição $\mathrm{I}+\mathrm{N}$ - cause um maior sentimento de presença que a condição I$\mathrm{N}+$. Especula-se também que existirá uma diferença no cybersickness entre ambos os jogos devido aos diferentes níveis de interação que requerem maior número de movimentos que podem entrar em conflito com o feedback visual recebido por parte do sistema de RV.

\section{ESTUDO EXPERIMENTAL}

Foram analisados dois jogos de RV disponíveis ao público através da plataforma STEAM (Software de gestão de direitos

TABELA I

JoGOS DE RV UTILIZADOS E O PESO DAS SUAS COMPONENTES DE INTERAÇÃO E NARRATIVA

\begin{tabular}{lll}
\hline \hline & Interação & Narrativa \\
\hline Endless Night (I+N-) & + & - \\
Quanero (I-N+) & - & +
\end{tabular}

digitais desenvolvido por Valve Corporation). O intuito deste estudo é perceber qual a influência do género e das componentes interação e narrativa nos jogos RV na elevação dos níveis de presença sentidos pelos jogadores e qual os seus possíveis efeitos no cybersickness. As experiências foram conduzidas sobre uma avaliação subjetiva. Ambos os jogos apresentam um nível de qualidade gráfica semelhante, o mesmo espaço físico onde o utilizador se pode mover e nenhum apresenta um corpo virtual representando o jogador, apenas as mãos são visíveis.

\section{A. Amostra}

Para cada jogo participaram 16 utilizadores, 8 mulheres e 8 homens, totalizando um total de 32 participantes. A idade mais alta situa-se nos 25 anos e a mais baixa nos 18 anos $(M=21$ $\mathrm{SD}=2,021)$, sendo eles maioritariamente estudantes universitários. 


\section{B. Instrumentos}

Para avaliação da sensação de presença, foi usada a versão portuguesa devidamente validada do questionário Igroup Presence Questionnaire (IPQp)[12][35]. Tal foi escolhido por ser um questionário validado na língua materna dos participantes. O questionário contém 14 itens numa escala de likert de cinco pontos com as seguintes 3 subescalas: presença espacial (a sensação de se encontrar fisicamente presente no ambiente virtual), realismo experienciado (mede a experiência subjetiva do realismo no ambiente virtual) e envolvimento (mede a atenção dada ao ambiente virtual e o nível de envolvimento experienciado). Para a avaliação do cybersickness foi usado o Simulator Sickness Questionnaire (SSQ) [27] traduzido para português. Este questionário foi escolhido por ser um dos mais usados atualmente na avaliação de cybersickness. O SSQ é constituído por 16 itens correspondentes a um conjunto de sintomas que podem ser classificados como: Nenhum, Ligeiro, Moderado e Severo. O $S S Q$ apresenta as seguintes 3 subescalas: náusea (náuseas, salivação, arrotos, estomago), desconforto oculomotor (dificuldade em focar, visão desfocada e cansaço ocular) e desorientação (tonturas e vertigens). Foi usado um questionário sociodemográfico com o objetivo de recolher dados genéricos sobre a amostra. O questionário continha questões sobre: género, idade e profissão.

\section{Materiais}

Para o estudo da sensação de presença e cybersickness, foram considerados jogos que focassem apenas numa das componentes (interação ou narrativa). Os critérios tidos em conta na escolha do jogo representativo da componente de interação tiveram em conta os conceitos de interação de Slater e Usoh, Zeltzer e Steuer [8], [18], [19]: capacidade do utilizador se deslocar, alterar o mundo virtual em tempo real e receber feedback dessas alterações.

Já na escolha do jogo representativo da componente narrativa, os critérios usados foram: o jogo deverá conter uma narrativa interessante e deverá requerer que o utilizador a entenda de maneira a passar de nível e/ou chegar ao fim deste. Com estes critérios em conta, a equipa de investigação, após uma análise a vários jogos de RV, optou por usar os jogos Endless Night, (focado na interação), e Quanero (focado na narrativa). Segue-se uma descrição mais detalhada de cada um:

1) Endless Night $(I+N-)$ : é um jogo de RV desenvolvido para os HTC Vive, disponibilizado na STEAM. Tem como produtora e desenvolvedora a Yerawizard $L L C$. Foi usada a versão alpha do jogo lançada a 6 de dezembro de 2016.

É um shooter baseado num sistema de rondas. O jogador começa dentro de uma casa com duas caçadeiras e duas pistolas em cima da mesa para escolha. As pistolas possuem maior precisão e menor dano, enquanto que as caçadeiras têm menor precisão mas maior dano sendo úteis apenas a pouca distância. É possível selecionar duas armas iguais ou uma de cada tipo. Após a sua seleção é necessário recarregá-las e disparar para a porta de saída. Ambas as armas recarregam automaticamente após pressionar um botão no comando. No entanto nas caçadeiras é preciso um movimento adicional vertical com o pulso ou braço para fazer encaixar os canos de volta. Após o disparo, o jogador é imediatamente colocado no meio da rua, num ambiente escuro acompanhado com som ambiente sombrio. Não é possível fugir para outra parte do mapa, permanecendo o jogador naquele espaço o jogo todo.

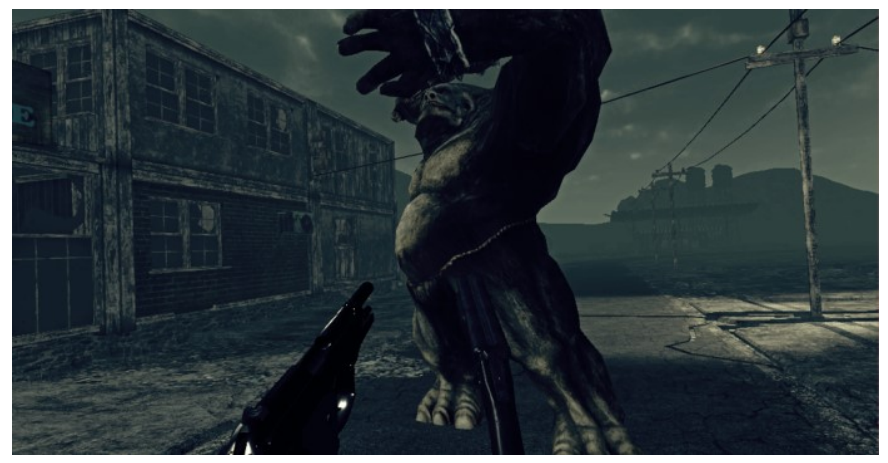

Fig. 1 Endless Night - Ojogador tenta matar um "troll" com uma caçadeira e uma pistola

Ronda após ronda a dificuldade vai aumentando com um aumento da diversidade e número de inimigos. Os monstros que o jogador enfrenta são:

- Pássaros de um olho que se vão aproximando do jogador pelo ar. Caso cheguem perto dele podem começar a lançar projeteis em forma de caveira às quais o jogador se pode desviar;

- Aranhas grandes que se tentam aproximar do jogador pelo chão;

- Bruxos que flutuam pelo ar e tentam aproximar-se do jogador;

- Trolls, criaturas de grande tamanho, lentos e difíceis de matar (Fig 1).

Dado que os inimigos aparecem vindos de todas as direções, via terrestre e aérea, o jogador necessita de manter uma atenção constante em todo o seu redor de maneira a não ser atacado pelas costas. A liberdade de movimento permitida leva a que o jogador possa desviar-se dos inimigos e projéteis. Uma maior atenção é requerida pelo facto de disparar uma arma em cada mão.

O dano sofrido pelo jogador é visível através de um filtro vermelho que aparece na imagem. Quanto maior é o dano mais vermelho se torna até perder o jogo. No fim é possível marcar as iniciais numa tabela de highscore. Após isso o jogador pode voltar a tentar desde o início, começando na sala inicial com as armas para escolha.

2) Quanero (I-N+) é uma experiência de RV criada por estudantes do programa de graduação da Universidade de Ciências Aplicadas Salzburg's MultiMediaArt and MultiMediaTechnology. Lançado a 12 de agosto de 2016 e disponibilizado na STEAM para HTC Vive e mais tarde Oculus Rift. Nesta experiência o jogador encontra-se num ambiente $S c i$ - $f$, num bar no topo de um prédio de uma cidade futurista (Fig. 2). Não é possível interagir diretamente com a história e 
elementos do jogo e as personagens não têm noção da presença do jogador. Este tem o poder de avançar, parar e recuar no tempo tal como de teletransportar-se para qualquer ponto do bar.

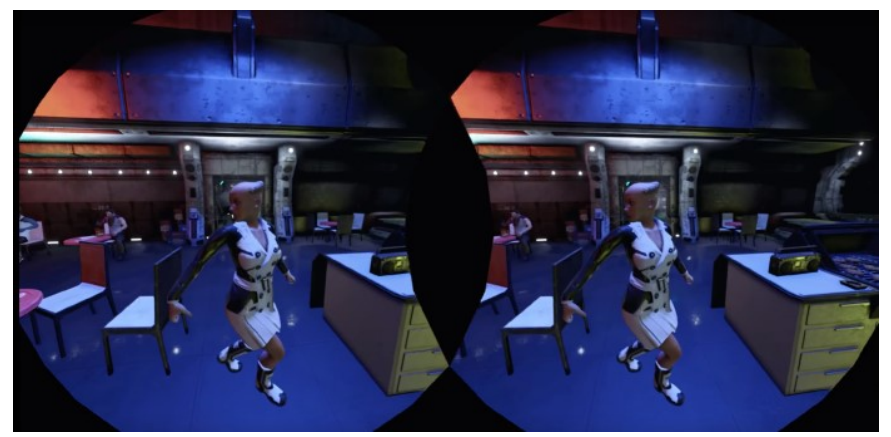

Fig. 2 Quanero - Zona Exterior

Uma série de acontecimentos em cadeia irão decorrer no local e o objetivo do jogador é tentar perceber essa cadeia de eventos e o que levou à causa de uma explosão. Várias personagens interagem entre si ao mesmo tempo. O jogador terá de usar os comandos para controlar o tempo e reviver a mesma janela temporal de diferentes locais e pontos de vista de maneira a descobrir o mistério atrás da dita explosão. Após reviver um certo momento, como por exemplo a explosão, uma memória é desbloqueada no menu e passa a ser possível reviver esse momento novamente e instantaneamente pelo menu.

O início do jogo oferece ao jogador um tutorial de como jogar antes de proceder à entrada no ambiente em questão. $\mathrm{O}$ bar é constituído por uma parte exterior e interior. No exterior encontra-se um homem a grelhar carne, um casal a discutir e dois homens, cada um sentado em mesas diferentes. No interior encontram-se um homem e uma mulher a jogar bilhar e mais dois homens sentados a conversar numa mesa. Existe também um pequeno robô que se desloca pelo chão que serve de aspirador.

A explosão decorre num grelhador no exterior e os eventos que o antecedem são os seguintes: Um dos homens sentado à mesa na zona exterior encontra-se a ler uma notícia num tablet sobre um suspeito de terrorismo. O indivíduo que se encontra na mesa ao lado a escrever numa folha de papel assemelha-se bastante à foto do suspeito. Numa outra mesa, um casal conversa normalmente até que o telemóvel do rapaz em cima da mesa vibra com uma chamada de outra pessoa, a partir daí começam a discutir. O homem que se encontra a grelhar carne repara que o grelhador deixou de funcionar e tenta descobrir a razão batendo nas botijas de gás. No interior, um homem e uma mulher jogam bilhar, enquanto numa mesa dois homens negoceiam os conteúdos de uma mala. Enquanto tudo isto acontece, o robô aspirador desloca-se ao pé do bar para apanhar uma febra de carne que tinha caído ao chão e segue para o interior do bar onde bate contra a perna da mulher que jogava bilhar. Esta engana-se na tacada e a bola sai disparada contra a cara do seu adversário que acaba por embater de costas contra um dos homens que estavam na mesa atrás. Ao colidir com este, um crachá da polícia cai do bolso do homem que estava sentado. Face a isto, o homem com quem o suposto polícia infiltrado estaria a negociar tira uma arma e tenta baleá-lo.

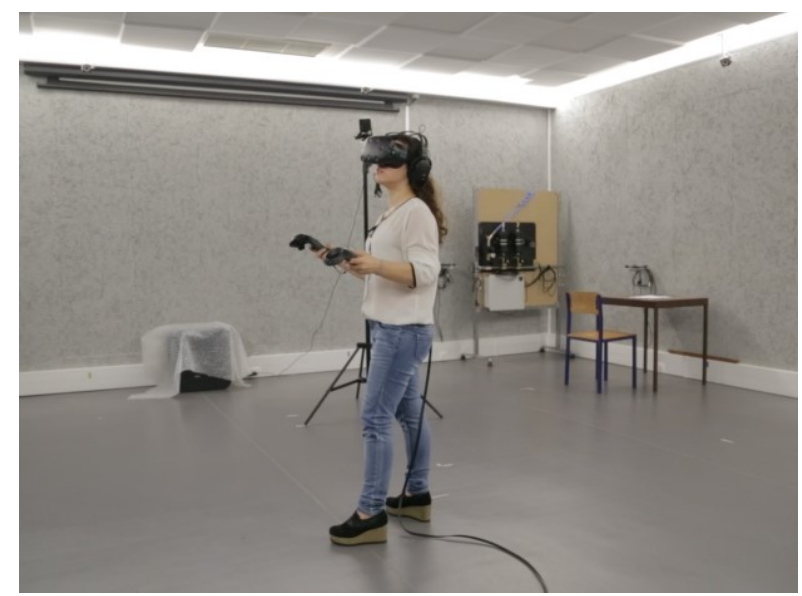

Fig. 3 Participante a realizar a experiência.

A bala falha e segue atravessando a parede, colidindo com as botijas de gás do grelhador no momento em que o cozinheiro estaria a dar um pontapé nelas, causando a bala efetivamente a explosão. Neste momento, o homem que estaria com o tablet sai de rompante e começa a lutar contra o indivíduo ao seu lado por pensar que a caneta que este usava para escrever numa folha era um detonador. A mulher do casal cai pelo buraco criado pela explosão, mas consegue assegurarse à berma com uma mão, o namorado pouco depois procede a puxá-la para cima.

Na narrativa deste jogo existem, portanto, várias histórias a acontecer ao mesmo tempo, algumas não ligadas à sequência de eventos que acabou por originar a explosão e outros indiretamente ligados à mesma. A função do jogador é, portanto, explorar todos os acontecimentos e chegar à resposta certa à pergunta "o que provocou a explosão".

Foram usados os Head Mounted Display (HMD) HTC Vive como dispositivo de visualização de RV e de tracking para todos os cenários experimentais cenários. A área de jogo pelo qual o participante se podia mover era de $2 \mathrm{~m} \times 2 \mathrm{~m}$. A resolução de ecrã dos $H M D$ eram de 2160x1200 com refresh rate de $90 \mathrm{hz}$ e campo de visão de $110^{\circ}$ (aprox). Juntamente foram utilizados dois comandos com tracking que o jogador usava para interagir com o ambiente virtual (Fig. 3). Para entrega de som foram usados headphones com cancelamento ruído de maneira a eliminar possíveis sons externos ao jogo.

Para a apresentação dos estímulos visuais e auditórios, foi utilizado um computador desktop com as seguintes características: $C P U$ i7-5820k @ 3.30GHz, 32GB de memória $R A M, 3$ Geforce GTX 980. O framerate estável e elevado garantiu que não houvesse influência do desempenho computacional nos resultados. 


\section{Variáveis do estudo}

As variáveis independentes definidas para este estudo foram o nível de interação e narrativa e o género dos participantes. As variáveis dependentes consideradas para a avaliação do sentimento de presença foram: presença, realismo experienciado, envolvimento e presença espacial. No que diz respeito ao sentimento de cybersickness, foram consideradas as escalas náusea, desconforto oculomotor, desorientação e cybersickness.

\section{E. Procedimento Experimental}

Antes de dar início à atividade experimental, foi explicado aos participantes o que iriam fazer. Cada participante assinou uma folha de consentimento e um questionário sociodemográfico. Após o preenchimento foi dado um pequeno briefing para explicar o objetivo do jogo e o funcionamento da plataforma HTC Vive em maior detalhe (comandos e movimentos que o jogador pode realizar dentro do espaço demarcado). O resto das instruções foram deixadas a cargo dos tutoriais que os jogos apresentavam. Caso os participantes não entendessem o inglês, eram explicadas as instruções dadas no tutorial antes de começar a jogar.

Os participantes jogaram até um máximo de 10 minutos (podendo ser a duração inferior caso cumprissem o objetivo do jogo mas nunca inferior a 5 minutos). A duração da experiência foi definida de modo a não prejudicar o sentimento de presença [25]. No fim foi solicitado o preenchimento do questionário de presença $(I P Q p)[35]$ e cybersickness $(S S Q)$ [27].

\section{RESUltados}

Inicialmente realizou-se uma normalização dos dados de forma a obter uma distribuição normal. Foi considerada uma amostra normal onde $\mid$ Assimetria $\mid<2$ e $\mid$ Curtose $\mid<2$. Foram encontrados 6 outliers que foram removidos ao descartar os dados desses mesmos 6 participantes. Após a sua remoção a Assimetria variou entre -0,537 e 1,518, e Curtose, entre -0,888 e 1,717. De maneira a analisar os efeitos das variáveis independentes (2 (interação/narrativa) x 2 (género)) foi usado uma análise multivariada de variância (MANOVA).

\section{A. Nivel de interação e narrativa}

Através da análise MANOVA os resultados obtidos mostram que não houve diferenças significativas entre as duas condições $\mathrm{I}+\mathrm{N}-$ e $\mathrm{I}-\mathrm{N}+$ relativamente à sensação de presença (Tabela II) $\left(\mathrm{F}(3,20)=0,233 ; \mathrm{p}>0,05 ;\right.$ Wilk's $\Lambda=0,966, \eta_{\mathrm{p}}{ }^{2}=$ $0,034 ; \mathrm{PO}=0,087)$ e cybersickness (Tabela III) $(\mathrm{F}(3,20)=$ 1,$890 ; \mathrm{p}>0,05 ;$ Wilk's $\left.\Lambda=0,779 ; \eta_{\mathrm{p}}^{2}=0,221 ; \mathrm{PO}=0,415\right)$. As médias das métricas de presença e cybersickness em ambas as condições podem ser visualizadas na Tabela V.

\section{B. Género}

$\mathrm{Na}$ análise MANOVA referente à variável independente Género encontrou-se uma diferença significativa para presença $\left(\mathrm{F}(3,20)=3,977 ; \mathrm{p}<0,05 ;\right.$ Wilk's $\Lambda=0,626 ; \eta_{\mathrm{p}}{ }^{2}=0,374 ; \mathrm{PO}=$ $0,752)$ (Tabela II) e cybersickness $(\mathrm{F}(3,20)=5,191 ; \mathrm{p}<0,05$; Wilk's $\left.\Lambda=0,562 ; \eta_{\mathrm{p}}{ }^{2}=0,438 ; \mathrm{PO}=0,864\right)$ (Tabela III). Foi realizada então uma análise de variância ANOVA para as subescalas de presença e cybersickness (Tabela IV). Foram
TABELA II - RESULTADOS DA ANÁLISE MULTIVARIADA DAS VARIÁVEIS INDEPENDENTES CORRESPONDENTES À SENSAC̃̃O DE PRESENCA

\begin{tabular}{lllll}
\hline \hline \multicolumn{1}{c}{ Variável Independente } & $\mathrm{F}$ & $\mathrm{P}$ & $\eta \mathrm{P}^{2}$ & $\mathrm{PO}$ \\
\hline Nível de interação e narrativa & 0,233 & 0,872 & 0,034 & 0,087 \\
Género & 3,977 & 0,023 & 0,374 & 0,752 \\
Nível de interação e narrativa & 3,545 & 0,033 & 0,347 & 0,698 \\
$\times$ Género & & & &
\end{tabular}

TABELA III - RESULTADOS DA ANÁLISE MULTIVARIADA DAS VARIÁVEIS INDEPENDENTES CORRESPONDENTES AO CYBERSICKNESS

\begin{tabular}{lllll}
\hline \hline \multicolumn{1}{c}{ Variável Independente } & $\mathrm{F}$ & $\mathrm{P}$ & $\eta \mathrm{P}^{2}$ & $\mathrm{PO}$ \\
\hline Nível de interação e narrativa & 1,890 & 0,164 & 0,221 & 0,415 \\
Género & 5,191 & 0,008 & 0,438 & 0,864 \\
Nível e interação e narrativa & 1,226 & 0,326 & 0,155 & 0,278
\end{tabular}

$\times$ Género

encontradas diferenças significativas na métrica de presença $(\mathrm{F}$ $\left.(1,22)=4,211 ; \mathrm{p}>0,05 ; \eta_{\mathrm{p}}{ }^{2}=0.161 ; \mathrm{PO}=0,501\right)$ apesar de $p=$ 0,052 podemos afirmar que estamos perante um erro tipo 2 pois houve um efeito forte $\left(\eta_{\mathrm{p}}{ }^{2}=0.161\right)$ e um $\mathrm{PO}=0,501$. O género feminino apresentou ter maiores índices de presença que o masculino. $\mathrm{O}$ mesmo aconteceu para realismo experienciado $\left(\mathrm{F}(1,22)=10,617 ; \mathrm{p}<0,05 ; \eta_{\mathrm{p}}{ }^{2}=0,325 ; \mathrm{PO}=\right.$ 0,876 ) onde o género feminino conseguiu maior pontuação. As médias das métricas de presença e cybersickness para cada um dos géneros podem ser verificados na Tabela $\mathrm{V}$.

Encontra-se o mesmo padrão nas métricas Náusea ( F $(1,22)$ $\left.=6,469 ; \mathrm{p}<0,05 ; \eta_{\mathrm{p}}{ }^{2}=0,227 ; \mathrm{PO}=0,681\right)$ e Desorientação $(\mathrm{F}$ $\left.(1,22)=4,869 ; \mathrm{p}<0,05 ; \eta_{\mathrm{p}}^{2}=0,181 ; \mathrm{PO}=0,560\right)$ com o género feminino a ter maiores índices que o masculino.

\section{Género vs. Nivel de Interação e Narrativa}

Analisando a combinação do nível de interação e narrativa com género foram encontradas diferenças significativas através da análise MANOVA nas métricas de presença (Tabela II) $\left(\mathrm{F}(3,20)=3,545, \mathrm{p}<0,05 ;\right.$ Wilk's $\Lambda=0,653, \eta_{\mathrm{p}}{ }^{2}=$ $0,347 ; \mathrm{PO}=0,698)$. Para cybersickness (Tabela III) não houve diferenças significativas $(\mathrm{F}(3,20)=1,226, \mathrm{p}>0,05$; Wilk's $\Lambda$ $\left.=0,845, \eta_{\mathrm{p}}^{2}=0,155 ; \mathrm{PO}=0,278\right)$.

\section{DISCUSSÃO}

Era expectável que um maior nível de interação nos jogos de RV provocaria uma maior sensação de presença do que um jogo com melhor narrativa, o que não se verificou. Os resultados obtidos relativamente à inexistência de diferenças significativas entre as duas condições $\mathrm{I}+\mathrm{N}-\mathrm{e} \mathrm{I}-\mathrm{N}+$ pode indicar que, de modo geral e dentro do conceito dos jogos de RV, ambos os componentes de interação e narrativa podem ter influências semelhantes na sensação de presença em geral. Isto

TABELA IV - ANÁLISE UNIVARIADA RELATIVA À VARIÁVEL INDEPENDENTE GÉNERO.

\begin{tabular}{lllll}
\hline \hline \multicolumn{1}{c}{ Escalas } & $\mathrm{F}$ & $\mathrm{P}$ & $\eta \mathrm{P}^{2}$ & $\mathrm{PO}$ \\
\hline Presença & 4,211 & 0,052 & 0,161 & 0,501 \\
Realismo experienciado & 10,617 & 0,004 & 0,325 & 0,876 \\
Envolvimento & 0,754 & 0,395 & 0,033 & 0,132 \\
Presença Espacial & 0,029 & 0,867 & 0,001 & 0,053 \\
Náusea & 6,469 & 0,019 & 0,227 & 0,681 \\
Desconforto oculomotor & 0,182 & 0,673 & 0,008 & 0,069 \\
Desorientação & 4,869 & 0,038 & 0,181 & 0,560 \\
Cybersickness & 3,078 & 0,093 & 0,123 & 0,389
\end{tabular}


TABELA V - MÉDIAS DAS MÉTRICAS DE PRESENÇA E CYBERSICKNESS ENTRE AS VARIÁVEIS INDEPENDENTES.

\begin{tabular}{lllll}
\hline \hline \multicolumn{1}{c}{ Escalas } & I+N- & I-N+ & Masc. & Femin. \\
\hline Presença & 3,549 & 3,599 & 3,438 & 3,760 \\
Realismo experienciado & 3,192 & 3,385 & 2,967 & 3,727 \\
Envolvimento & 3,615 & 3,519 & 3,433 & 3,750 \\
Presença Espacial & 3,744 & 3,795 & 3,756 & 3,788 \\
Náusea & 8,806 & 5,137 & 4,452 & 10,407 \\
Desconforto oculomotor & 11,078 & 4,082 & 7,075 & 8,269 \\
Desorientação & 14,991 & 5,354 & 5,568 & 16,451 \\
Cybersickness & 12,946 & 5,466 & 6,732 & 12,580
\end{tabular}

poderá indicar que o mesmo nível de sensação de presença pode ser atingido tanto por uma boa narrativa como com um nível alto de interação.

No cybersickness também não foram encontradas diferenças entre jogos. Apesar do jogo que envolve uma maior interação exigir bastantes movimentos corporais com vários elementos a deslocar-se no ambiente virtual, este não provocou mais cybersickness que o jogo representativo da narrativa. $\mathrm{O}$ cybersickness geralmente surge quando o sistema vestibular entra em conflito com a visão [9], ou seja, quando o que estamos a ver não coincide com o movimento que estamos a sentir e vice-versa. O facto de não ter existido diferença no cybersickness entre os jogos pode sugerir que o equipamento de RV usado evoluiu de maneira a conseguir acompanhar coerentemente os vários movimentos corporais e dar o devido feedback visual. Portanto um maior nível de interação em si não deverá provocar sintomas de cybersickness relativamente a um jogo com interação mais passiva.

No que toca à variável independente género foram encontradas diferenças significativas na sensação de presença nas métricas presença e realismo experienciado e no cybersickness nas métricas náusea e desorientação com o género feminino a ter pontuações mais altas que o masculino. Estes resultados não corroboram o estudo de Narciso et al. [26], onde não foram registadas diferenças. Tal pode dever-se ao facto de o estudo não envolver interação e por se tratar de vídeos $360^{\circ}$ invés de ambientes virtuais $3 \mathrm{D}$. Na combinação do nível de interação e narrativa com género encontraram-se diferenças significativas na presença.

Estes resultados na sensação de presença entre géneros contrastam com outro estudo onde o género masculino foi quem teve maiores índices de presença [22] [23]. Em concordância com os resultados obtidos, um estudo realizado num sistema pouco imersivo para comparar a sensação de presença com a qualidade de imagem nos ecrãs HDTV demonstrou uma diferença significativa entre géneros onde o feminino apresentou maior realismo experienciado em relação ao género masculino [24].

Existem limitações neste estudo tais como: a amostra não representa com rigor a população em geral, sendo esta toda constituída por estudantes entre os 18 e 25 anos de idade. Apesar de parecidos em termos de grafismo, os jogos diferem em muitos aspetos para além da interação e narrativa, tais como som ambiente ou género do jogo (ex. ação, suspense, aventura, arcade). $\mathrm{O}$ cybersickness parece estar também correlacionado positivamente com a sensação de presença [33] não sendo possível determinar com certeza se este nível de cybersickness é devido à diferença de género ou ao aumento da sensação de presença. É preciso ter em atenção igualmente que a experiência prévia com ambientes interativos pode influenciar os níveis de presença [23]. Apesar desta experiência prévia com jogos costumar estar associada em geral ao género masculino [36], na amostra utilizada para este estudo poderá ter influenciado os resultados.

Como trabalho futuro é necessário juntar a este estudo um jogo que combine um alto nível de interação e uma boa narrativa e analisar se a combinação destas duas componentes resultará numa maior sensação de presença do que aquela apresentada nas duas condições estudas neste trabalho. É preciso verificar outros fatores que contribuam para a diferença nas métricas de presença e cybersickness entre géneros. Possíveis fatores a serem estudados podem ser a existência de uma utilização prévia de equipamentos de RV, a frequências com que os participantes jogam, os traços de personalidade, o conhecimento da linguagem usada (inglês) e o nível de conhecimento do funcionamento da RV.

\section{CONCLUSÃO}

Este estudo pretendeu analisar a influência que as componentes de interação e narrativa e o género têm na sensação de presença e cybersickness. Foi estudado como um jogo com muita interação e pouca narrativa e outro com pouca interação e boa narrativa afetam a presença e cybersickness. Não foram encontradas diferenças significativas nestas variáveis dependentes quando comparando os dois jogos. Isto pode indicar que ambas as componentes interação e narrativa têm impactos semelhantes na sensação de presença e que um maior nível de interação não influência o cybersickness. Como trabalho futuro é necessário analisar um jogo que inclua tanto um nível alto de interação como uma boa narrativa.

\section{AGRADECIMENTOS}

Este trabalho foi financiado pelo Projeto CHIC N. ${ }^{\circ} 24498$, financiado por Fundo Europeu de Desenvolvimento Regional (FEDER) através do COMPETE 2020 - Programa Operacional Competitividade e Internacionalização (POCI). Todos os trabalhos foram desenvolvidos no Laboratório de Realidade Virtual MASSIVE.

\section{REFERENCIAS BIBLIOGRÁFICAS}

[1] P. Fuchs, G. Moreau, and P. Guitton, Eds., Virtual reality: concepts and technologies. Boca Raton, FL: CRC Press, 2011.

[2] P. Fuchs, Les interfaces de la réalité virtuelle. éditeur AJIIMD, 1996.

[3] R. M. Baños, C. Botella, M. Alcañiz, V. Liaño, B. Guerrero, and B. Rey, "Immersion and Emotion: Their Impact on the Sense of Presence," Cyberpsychol. Behav., vol. 7, no. 6, pp. 734-741, Dec. 2004.

[4] M. Meehan, B. Insko, M. Whitton, and F. P. Brooks, "Physiological measures of presence in stressful virtual environments," 2002, p. 645. 
[5] H. S. Wallach, M. P. Safir, and R. Samana, "Personality variables and presence," Virtual Real., vol. 14, no. 1, pp. 3-13, Mar. 2010.

[6] M. Slater and S. Wilbur, "A Framework for Immersive Virtual Environments (FIVE): Speculations on the Role of Presence in Virtual Environments," Presence Teleoperators Virtual Environ., vol. 6, no. 6, pp. 603616, Dec. 1997.

[7] M. Slater, M. Usoh, and A. Steed, "Depth of Presence in Virtual Environments," Presence Teleoperators Virtual Environ., vol. 3, no. 2, pp. 130-144, Jan. 1994.

[8] J. Steuer, "Defining Virtual Reality: Dimensions Determining Telepresence," J. Commun., vol. 42, no. 4, pp. 73-93, Dec. 1992.

[9] J. J. LaViola, "A discussion of cybersickness in virtual environments," ACM SIGCHI Bull., vol. 32, no. 1, pp. 47-56, Jan. 2000.

[10] R. Baños, C. Botella, V. Liaño, B. Guerrero, B. Rey, and M. Alcañiz, "Sense of presence in emotional virtual environments," Proc. Presence, pp. 156-159, 2004.

[11] B. G. Witmer and M. J. Singer, "Measuring Presence in Virtual Environments: A Presence Questionnaire," Presence Teleoperators Virtual Environ., vol. 7, no. 3, pp. 225-240, Jun. 1998.

[12] T. Schubert, F. Friedmann, and H. Regenbrecht, "The Experience of Presence: Factor Analytic Insights," Presence Teleoperators Virtual Environ., vol. 10, no. 3, pp. 266-281, Jun. 2001.

[13] J. Lessiter, J. Freeman, E. Keogh, and J. Davidoff, “A Cross-Media Presence Questionnaire: The ITC-Sense of Presence Inventory," Presence Teleoperators Virtual Environ., vol. 10, no. 3, pp. 282-297, Jun. 2001.

[14] I. Messinis, D. Saltaouras, P. Pintelas, and T. Mikropoulos, "Investigation of the Relation Between Interaction and Sense of Presence in Educational Virtual Environments," 2010, pp. 428-431.

[15] R. P. McMahan, D. A. Bowman, D. J. Zielinski, and R. B. Brady, "Evaluating Display Fidelity and Interaction Fidelity in a Virtual Reality Game," IEEE Trans. Vis. Comput. Graph., vol. 18, no. 4, pp. 626-633, Apr. 2012.

[16] M. Slater, "Place illusion and plausibility can lead to realistic behaviour in immersive virtual environments," Philos. Trans. R. Soc. B Biol. Sci., vol. 364, no. 1535, pp. 3549-3557, Dec. 2009.

[17] R. B. Welch, T. T. Blackmon, A. Liu, B. A. Mellers, and L. W. Stark, "The Effects of Pictorial Realism, Delay of Visual Feedback, and Observer Interactivity on the Subjective Sense of Presence," Presence Teleoperators Virtual Environ., vol. 5, no. 3, pp. 263-273, Jan. 1996.

[18] M. Slater and M. Usoh, "Body centred interaction in immersive virtual environments," Artif. Life Virtual Real., vol. 1, pp. 125-148, 1994.

[19] D. Zeltzer, "Autonomy, Interaction, and Presence," Presence Teleoperators Virtual Environ., vol. 1, no. 1, pp. 127-132, Jan. 1992.

[20] E. Brown and P. Cairns, "A grounded investigation of game immersion,” 2004, p. 1297.
[21] R. Busselle and H. Bilandzic, "Measuring Narrative Engagement," Media Psychol., vol. 12, no. 4, pp. 321347, Nov. 2009.

[22] A. Felnhofer, O. D. Kothgassner, L. Beutl, H. Hlavacs, and I. Kryspin-Exner, "Is virtual reality made for men only? Exploring gender differences in the sense of presence," Proc. Int. Soc. Presence Res., pp. 103-112, 2012.

[23] K. Lachlan and M. Krcmar, "Experiencing Presence in Video Games: The Role of Presence Tendencies, Game Experience, Gender, and Time Spent in Play," Commun. Res. Rep., vol. 28, no. 1, pp. 27-31, Feb. 2011.

[24] C. C. Bracken, "Presence and Image Quality: The Case of High-Definition Television,” Media Psychol., vol. 7, no. 2, pp. 191-205, May 2005.

[25] M. Melo, J. Vasconcelos-Raposo, and M. Bessa, "Presence and cybersickness in immersive content: Effects of content type, exposure time and gender," Comput. Graph., vol. 71, pp. 159-165, Apr. 2018.

[26] D. Narciso, M. Bessa, M. Melo, A. Coelho, and J. Vasconcelos-Raposo, "Immersive $360^{\circ}$ video user experience: impact of different variables in the sense of presence and cybersickness," Univers. Access Inf. Soc., Oct. 2017.

[27] R. S. Kennedy, N. E. Lane, K. S. Berbaum, and M. G. Lilienthal, "Simulator Sickness Questionnaire: An Enhanced Method for Quantifying Simulator Sickness," Int. J. Aviat. Psychol., vol. 3, no. 3, pp. 203-220, Jul. 1993.

[28] A. Graybiel, C. D. Wood, E. F. Miller, and D. B. Cramer, "Diagnostic criteria for grading the severity of acute motion sickness," Aerosp. Med., vol. 39, no. 5, pp. 453455, May 1968.

[29] S. Davis, K. Nesbitt, and E. Nalivaiko, “A Systematic Review of Cybersickness," 2014, pp. 1-9.

[30] K. M. Stanney and P. Hash, "Locus of User-Initiated Control in Virtual Environments: Influences on Cybersickness," Presence Teleoperators Virtual Environ., vol. 7, no. 5, pp. 447-459, Oct. 1998.

[31] F. Biocca, "Will Simulation Sickness Slow Down the Diffusion of Virtual Environment Technology?," Presence Teleoperators Virtual Environ., vol. 1, no. 3, pp. 334-343, Jan. 1992.

[32] J. T. Reason and J. J. Brand, Motion Sickness. Academic Press, 1975.

[33] J. J. W. Lin, H. B. L. Duh, D. E. Parker, H. Abi-Rached, and T. A. Furness, "Effects of field of view on presence, enjoyment, memory, and simulator sickness in a virtual environment," in Proceedings IEEE Virtual Reality 2002, 2002, pp. 164-171.

[34] P. Zahorik and R. L. Jenison, "Presence as Being-in-theWorld," Presence Teleoperators Virtual Environ., vol. 7, no. 1, pp. 78-89, Feb. 1998.

[35] J. Vasconcelos-Raposo et al., "Adaptation and Validation of the Igroup Presence Questionnaire (IPQ) in a Portuguese Sample," Presence Teleoperators Virtual Environ., vol. 25, no. 3, pp. 191-203, Dec. 2016. 
[36] T. Hartmann and C. Klimmt, "Gender and Computer Mediat. Commun., vol. 11, no. 4, pp. 910-931, Jul. 2006. Games: Exploring Females’ Dislikes," J. Comput.- 